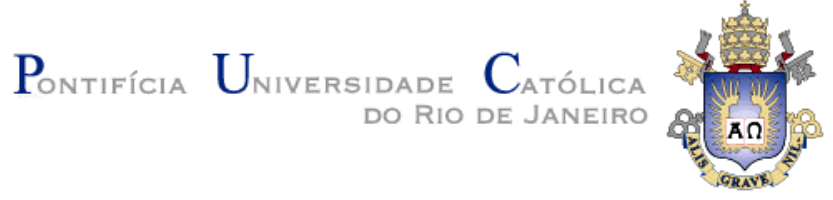

Katia Fabiola Cánepa Vega

\title{
TREG: Un juego de entrenamiento en Ingeniería de Requisitos
}

\section{DISSERTAÇÃO DE MESTRADO}

Tesis presentada como requisito parcial para obtener el título de Mestre por el programa de Pos-Graduación en Informática de la PUC-Rio.

Orientador: Prof. Hugo Fuks Co-orientador: Gustavo Robichez de Carvalho 


\section{TREG: Un juego de entrenamiento en Ingeniería de Requisitos}

Tesis presentada como requisito parcial para obtener el título de Magíster por el programa de Pos-Graduación en Informática de la PUC-Rio. Aprobada por la Comisión Examinadora que suscribe a continuación.

Prof. Hugo Fuks

Orientador

Departamento de Informática - PUC-Rio

Gustavo Robichez de Carvalho

Co-orientador

Departamento de Informática - PUC-Rio

Prof. Alberto Barbosa Raposo

Departamento de Informática - PUC-Rio

Mariano Pimentel

UNIRIO

Prof. José Eugenio Leal Coordinador(a) Sectorial del Centro Técnico Científico - PUC-Rio 
All rights reserved

\section{Katia Fabiola Cánepa Vega}

Graduada en Ingeniería de Sistemas e Informática por la Universidad Nacional Mayor de San Marcos (UNMSM), Perú. Su área de investigación actual es mundos virtuales, juegos y colaboración. Trabajó como consultora de procesos de desarrollo de software y de las herramientas de IBM Rational. Desarrolló, como proyecto de maestría, un juego para el entrenamiento en ingeniería de requisitos.

Datos Bibliográficos

Cánepa Vega, Katia Fabiola

TREG : un juego de entrenamiento en ingeniería de requisitos / Katia Fabiola Cánepa Vega ; orientador: Hugo Fuks ; co-orientador: Gustavo Robichez de Carvalho. - 2010.

133 f. : il.(color.) ; $30 \mathrm{~cm}$

Dissertação (Mestrado)-Pontifícia Universidade Católica do Rio de Janeiro, Departamento de Informática, Rio de Janeiro, 2010.

Inclui bibliografia

1. Informática - Teses. 2. Jogo. 3. Prototipação. 4. CSCL. 5. Second Life. 6. Engenharia de requisitos. I. Fuks, Hugo. II. Carvalho, Gustavo Robichez de. III. Pontifícia Universidade Católica do Rio de Janeiro. Departamento de Informática. IV. Título. 
A mis padres,

Carlos y Lida,

por su amor infinito 


\section{Agradecimientos}

A mi asesor, Profesor Hugo Fuks, por confiar en mí, por ser un ejemplo constante de investigador y por, entre muchas otras palabras, enseñarme el significado de osadía.

A mi co-asesor, Gustavo Robichez, por su dedicación y siempre valiosas sugerencias que me ayudaron a analizar las cosas desde varias perspectivas.

A la banca examinadora por sus importantes contribuciones en el refinamiento de este trabajo.

A mis colegas de Groupware del LES, por su compañerismo a lo largo de estos dos años; en especial a Bruno y Andréia por sus demostraciones de colaboración y amistad.

A mis padres Lida y Carlos, por todo su esfuerzo en hacerme crecer y por su amor infinito y sin fronteras. A mis hermanos Renatto y Carlos, y a mi tía Mary por sus demostraciones de cariño e incentivo en seguir adelante.

A Paola, Cris, Ugo, Silvinha y Elizabeth por convertirse en mi familia en este otro lado del continente. A mis amigos, Diego, Maribel, Maju, Geraldine, Mila, por enseñarme lo que es la amistad verdadera.

A la PUC-Rio por acogerme en esta mi nueva casa de estudios y a la agencia CAPES por el apoyo financiero para la realización de este proyecto. 


\section{Resumen}

Cánepa Vega, Katia Fabiola; Fuks, Hugo. TREG: Un juego de entrenamiento en Ingeniería de Requisitos. Rio de Janeiro, 2010. 133p. Tesis de Maestría - Departamento de Informática, Pontifícia Universidade Católica do Rio de Janeiro.

TREG es un juego para el entrenamiento en Ingeniería de Requisitos específicamente en la técnica de Workshops. Fue creado en Second Life, usando las posibilidades de modelamiento 3D y programación en este ambiente. Este trabajo presenta una experiencia de exploración en un proceso de prototipación y técnicas para desarrollar el juego. El ciclo de desarrollo de TREG utiliza el proceso de prototipación basado en el libro "Effective Prototyping for Software Makers", este es un proceso iterativo que fue personalizado para la creación del juego educacional en este mundo virtual. Branching Stories es un género de simulaciones que da una visión global de todos los caminos posibles del jugador en TREG. La técnica de Escenarios es usada para la especificación de las simulaciones modeladas en el grafo de Branching Stories. El diseño de TREG utiliza las especificaciones realizadas con la técnica de escenarios para modelar diversas perspectivas del software. Las Maquinas de Estados modelan el comportamiento dinámico de los elementos del software, el diagrama de clases representa los objetos creados en Second Life y el diagrama de comunicación muestra las relaciones y paso de mensajes entre los objetos. Además, este trabajo muestra los resultados de la evaluación realizada así como las dificultades presentadas por los participantes al utilizar esta herramienta.

\section{Palabras clave}

Juego, prototipación, CSCL, Second Life, Ingeniería de Requisitos. 


\section{Resumo}

Cánepa Vega, Katia Fabiola; Fuks, Hugo. TREG: Um jogo para o treinamento em Engenharia de Requisitos. Rio de Janeiro, 2010. 133p. Dissertação de Mestrado - Departamento de Informática, Pontifícia Universidade Católica do Rio de Janeiro.

TREG é um jogo para o treinamento em Engenharia de Requisitos, especificamente na técnica de Workshops. Foi criado no Second Life, usando suas possibilidades de modelagem 3D e programação. Este trabalho apresenta uma experiência de exploração num processo de prototipação e técnicas para o desenvolvimento do jogo. O ciclo de desenvolvimento do TREG utiliza o processo de prototipação baseado no livro "Effective Prototyping for Software Makers", que descreve um processo iterativo que foi customizado para a criação do jogo educacional neste mundo virtual. Branching Stories é um género de simulações que dá uma visão geral de todos os caminhos possíveis do jogador no TREG. A técnica de Cenários é utilizada para a especificação das simulações modeladas no gráfico de Branching Stories. O desenho do TREG utiliza especificações feitas usando a técnica de Cenários para a modelagem de diferentes perspectivas de software. As maquinas de Estados modelam 0 comportamento dinâmico dos elementos de software, o diagrama de classes representa os objetos criados no Second Life e o diagrama de comunicação mostra as relações e a passagem de mensagens entre os objetos. Além disso, este trabalho apresenta os resultados dos testes de usabilidade e as dificuldades apresentadas pelos participantes para a utilização desta ferramenta.

\section{Palavras chave}

Jogo, prototipação, CSCL, Second Life, Engenharia de Requisitos. 


\section{Abstract}

Cánepa Vega, Katia Fabiola; Fuks, Hugo. TREG: A game for training in Requirements Engineering. Rio de Janeiro, 2010. 133p. MSc. Dissertation - Departamento de Informática, Pontifícia Universidade Católica do Rio de Janeiro.

TREG is a game for training in Requirements Engineering, specifically in the Workshops technique. It was created in Second Life using its building and scripting possibilities. This works presents an exploration in the use of a prototyping process and techniques for developing the game. The prototyping process of the book "Effective Prototyping for Software Makers" is an iterative process which was customized for the development of the game in this virtual world. Branching Stories is a simulation genre that gives an overview of all the possible paths the player can take in TREG. Scenario is a Requirements Engineering technique used for the specification of the simulations modeled in the Branching Stories graph. The design of TREG used the Scenarios specifications for modeling the software perspectives. The state machine diagrams shows the dynamic behavior of the TREG objects, a class diagram represents the objects created in Second Life and the communication diagrams shows the relationships and the flow of messages between objects. This work also shows the results of the evaluation made and the difficulties presented by the participants whereas TREG was used.

\section{Keywords}

Game, prototyping, CSCL, Second Life, Requirements Engineering. 


\section{Tabla de contenido}

1 Introducción 14

1.1. Motivación 14

1.2. Definición del proyecto 16

$\begin{array}{ll}\text { 1.2.1. Objetivos } & 16\end{array}$

1.2.2. Alcance 16

$\begin{array}{lr}\text { 1.2.3. Cronograma del proyecto } & 17\end{array}$

2 Marco teórico 18

$\begin{array}{lr}\text { 2.1. Mundos Virtuales } & 18\end{array}$

2.1.1. Second Life 19

2.1.2. Machinima 19

2.2. Workshops: Técnicas en Ingeniería de Requisitos 20

2.3. Técnicas para el desarrollo del juego 21

2.3.1. Proceso de prototipación 22

2.3.2. Branching Stories $\quad 22$

2.3.3. Escenarios 23

2.3.4. Diagramas de clases $\quad 24$

2.3.5. Diagramas de comunicación 25

2.3.6. Maquinas de estados 25

2.4. Trabajos relacionados 26

3 Características y aspectos del desarrollo de TREG 28

3.1. Características del juego 28

3.2. Contenido Educacional 31

3.2.1. Personas Adecuadas 32

3.2.2. Propósito Compartido 33

3.2.3. Trabajo Previo 34

3.2.4. Espacio Compartido 36

3.3. Diseño de TREG 37

3.3.1. Branching Stories en TREG 38

3.3.2. Escenarios de TREG 39

3.3.3. Modelamiento de TREG 42 
4 Prototipos $\quad 49$

4.1. Proceso de prototipación $\quad 49$

4.1.1. Fase 1. Planificar 49

4.1.2. Fase 2: Especificar 50

4.1.3. Fase 3. Diseñar 50

4.1.4. Fase 4. Resultados 51

4.2. Características de los prototipos de TREG 52

4.3. Criterios de Diseño 54

4.4. Iteraciones $\quad 57$

4.4.1. Iteración 1.

4.4.2. Iteración 2.

4.4.3. Iteración $3 . \quad 60$

4.4.4. Iteración $4 . \quad 61$

4.4.5. Iteración 5.

5 Pruebas y análisis de resultados 63

5.1. Propósito 63

5.1.1. Aspectos de la evaluación 63

5.1.2. Participantes 65

5.2. Desarrollo de las pruebas 66

5.2.1. Localización y condiciones técnicas 66

5.2.2. Acuerdos 66

5.2.3. Perfil de los participantes $\quad 67$

5.2.4. Entrenamiento en Second Life $\quad 67$

5.2.5. Durante las pruebas 68

5.2.6. Después de las pruebas $\quad 68$

5.3. Resultados 74

5.3.1. Interfaz 75

5.3.2. Mecanismos $\quad 77$

5.3.3. Jugabilidad 79

6 Conclusiones y trabajos futuros 82

6.1. Conclusiones 82

6.2. Trabajos futuros 85

7 Referencias Bibliográficas $\quad 87$ 
Apéndice A - Diseño del software $\quad 90$

A.1. Escenario: Personas Adecuadas. Excepción opción A 90

A.2. Escenario: Personas Adecuadas. Excepción opción B 91

A.3. Escenario: Personas Adecuadas. Excepción opción D 92

A.4. Canales de comunicación de TREG 93

A.5. Ejemplos de diagramas de comunicación en TREG 96

$\begin{array}{ll}\text { A.6. Ejemplos de diagramas de estados en TREG } & 97\end{array}$

Apéndice B - Documentos usados en las pruebas de usabilidad 98

B.1. Término de Consentimiento 98

$\begin{array}{ll}\text { B.2. Cuestionario del Perfil y Experiencia del Participante } & 100\end{array}$

B.3. Entrenamiento en Second Life para el uso de TREG 101

$\begin{array}{ll}\text { B.4 Entrevista } & 105\end{array}$

$\begin{array}{lr}\text { B5 Cuestionario IPQ } & 107\end{array}$

B6. Detalle del entrenamiento en Second Life 109

$\begin{array}{ll}\text { B7. Resultados del Cuestionario IP } & 110\end{array}$

$\begin{array}{ll}\text { B8. Resultados del Cuestionario IPQ } & 111\end{array}$

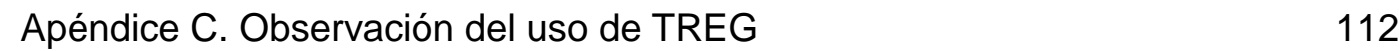

$\begin{array}{ll}\text { C1. Reporte de las Pruebas de Usabilidad de TREG (P2) } & 113\end{array}$

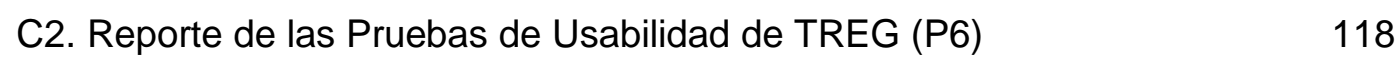

C3. Reporte de las Pruebas de Usabilidad de TREG (P8) 123

Apéndice D. Capturas de pantallas $\quad 128$

$\begin{array}{ll}\text { D.1. Áreas de TREG } & 128\end{array}$

$\begin{array}{lr}\text { D.2. Panel de Simulación } & 129\end{array}$

$\begin{array}{ll}\text { D.3. Ingrediente de Personas Adecuadas } & 130\end{array}$

$\begin{array}{ll}\text { D.4. Ingrediente de Propósito Compartido } & 131\end{array}$

$\begin{array}{ll}\text { D.5. Ingrediente de Trabajo Previo } & 132\end{array}$

$\begin{array}{ll}\text { D.6. Ingrediente de Espacio Compartido } & 133\end{array}$ 


\section{Lista de figuras}

Figura 1 Cronograma de actividades de TREG 17

Figura 2 The Effective Prototyping Process de [9]. 22

Figura 3 Estructura de Branching Stories de [5]. 23

Figura 4 Escenario del Ingrediente Personas adecuadas. 24

Figura 5 Diagrama de estados de uno de los elementos de TREG 26

Figura 6 A Groupthink game (A). Hospital Scenario en Play2Train (B) 27

Figura 7 La metáfora de la cocina y el cuarto de simulaciones de Workshops. 29

Figura 8 Miss Workshop, el NPC guía. 30

Figura 9 Funcionamiento de TREG desde la perspectiva del jugador. $\quad 30$

Figura 10 Distribución de los ingredientes en el proceso de Workshops $\quad 31$

Figura 11. Iteraciones de un Workshops. Trabajo Previo de [8] 35

Figura 12. Dimensiones de espacio y tiempo en las reuniones [8]. 36

Figura 13 Proceso de Diseño de TREG 37

Figura 14. Grafo de Branching Stories de TREG. 38

Figura 15. Escenario WS03 del ingrediente de Personas Adecuadas 40

Figura 16. Diagrama de clases para representar los elementos de TREG 43

Figura 17. Diagrama de Comunicación del Escenario WS03 45

Figura 18. Maquinas de estados de un NPC en Personas Adecuadas $\quad 47$

Figura 19. Implementación de estados de NPC de Personas Adecuadas $\quad 47$

Figura 20. Prototipos desarrollados en TREG 58

Figura 21. Prototipo de la Iteración 1.

Figura 22. Prototipo de la Iteración 2. 60

Figura 23. Prototipo de la Iteración 3.

Figura 24. Prototipo de la Iteración 4.

Figura 25. Prototipo de la Iteración 5.

Figura 26. Respuestas al cuestionario IPQ 81

Figura A.1. Escenario de Personas Adecuadas. Excepción opción A 90

Figura A.2. Escenario de Personas Adecuadas. Excepción opción B 91

Figura A.3. Escenario de Personas Adecuadas. Excepción opción D 92

Figura A.4. Diagrama de comunicación del Escenario WS04 96

Figura A.5. Diagrama de comunicación del Escenario WS04 96 


\section{Lista de tablas}

Tabla 1. Pros y contras de los posibles participantes al workshop 33

Tabla 2. Participantes al workshop y sus características 41

Tabla 3. Iteraciones y sus características 52

Tabla 4. Funciones con mayor retraso (en segundos) 57

Tabla 5. Aspectos a evaluar del juego 64

Tabla 6. Perfil de los participantes en las pruebas. 67

Tabla 7 Uso de Second Life $\quad 69$

Tabla 8 Facilidad de uso de los ambientes en TREG 69

Tabla 9 Interacción con los elementos del juego (Panel de Simulación) 70

Tabla 10 Facilidad de uso de los ingredientes $\quad 71$

Tabla 11 Entendimiento del objetivo del juego. (Personas Adecuadas) 72

Tabla 12. Elementos del cuestionario IPQ. 73

Tabla 13 Tiempo empleado en el juego 74

Tabla 14 Número de Intentos empleados en cada tarea de TREG 75

Tabla 15 Resultados de la entrevista. Interacción con los elementos del juego 75

Tabla 16. Observaciones más comunes en la interacción con Second Life 76

Tabla 17. Observaciones más comunes en la interacción con los elementos de $\begin{array}{ll}\text { TREG } & 76\end{array}$

Tabla 18 Resultados de la entrevista. Facilidad de Uso 77

Tabla 19 Reincidencias en la facilidad de Uso 78

Tabla 20 Resultados de la entrevista. Entendimiento del objetivo 79

Tabla 21 Reincidencias en el entendimiento del objetivo del juego 80

Tabla 22. Conversión de resultados del cuestionario IPQ 80

Tabla 23. Promedio de los factores de Presencia del cuestionario IPQ 81

Tabla A.1. Canales de comunicación de TREG 93

Tabla B.1. Respuestas al cuestionario IPQ 109

Tabla B.2. Detalle de intentos de los participantes $\quad 110$

Tabla B.3. Detalle del tiempo empleado por los participantes 110

Tabla B.4. Respuestas al cuestionario IPQ 111 